October 31, 2018

\title{
TOPOLOGICAL ASPECTS OF DIFFERENTIAL CHAINS
}

\author{
J. HARRISON \\ DEPARTMENT OF MATHEMATICS \\ UNIVERSITY OF CALIFORNIA, BERKELEY \\ H. PUGH \\ DEPARTMENT OF PURE MATHEMATICS AND MATHEMATICAL STATISTICS \\ UNIVERSITY OF CAMBRIDGE
}

\begin{abstract}
In this paper we investigate the topological properties of the space of differential chains $' \mathcal{B}(U)$ defined on an open subset $U$ of a Riemannian manifold $M$. We show that ${ }^{\prime} \mathcal{B}(U)$ is not generally reflexive, identifying a fundamental difference between currents and differential chains. We also give several new brief (though non-constructive) definitions of the space ${ }^{\prime} \mathcal{B}(U)$, and prove that it is a separable ultrabornological $(D F)$-space.

Differential chains are closed under dual versions of fundamental operators of the Cartan calculus on differential forms [10] 9]. The space has good properties some of which are not exhibited by currents $\mathcal{B}^{\prime}(U)$ or $\mathcal{D}^{\prime}(U)$. For example, chains supported in finitely many points are dense in ${ }^{\prime} \mathcal{B}(U)$ for all open $U \subset M$, but not generally in the strong dual topology of $\mathcal{B}^{\prime}(U)$.
\end{abstract}

\section{INTRODUCTION}

We begin with a Riemannian manifold $M$. Let $U \subset M$ be open and $\mathcal{P}_{k}=\mathcal{P}_{k}(U)$ the space of finitely supported sections of the $k$-th exterior power of the tangent bundle $\Lambda_{k}(T U)$. Elements of $\mathcal{P}_{k}(U)$ are called pointed $k$-chains in $U$. Let $\mathcal{F}=\mathcal{F}(U)$ be a complete locally convex space of differential forms defined on $U$. We find a predual to $\mathcal{F}$, that is, a complete l.c.s. ' $\mathcal{F}$ such that $\left({ }^{\prime} \mathcal{F}\right)^{\prime}=\mathcal{F}$. The predual

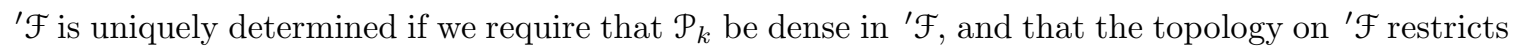
to the Mackey topology on $\mathcal{P}_{k}$, the finest locally convex topology on $\mathcal{P}_{k}$ such that $\left({ }^{\prime} \mathcal{F}\right)^{\prime}=\mathcal{F}$. Two natural questions arise: (i) Is ' $\mathcal{F}$ reflexive? (ii) Is there a constructive definition of the topology on 'F?

Let $\varepsilon_{k}$ be the space of $C^{\infty} k$-forms defined on $U$, and $\mathcal{D}_{k}$ the space of $k$-forms with compact support in $U$. Then $\mathcal{D}_{k}$ is an $(L F)$-space, an inductive limit of Fréchet spaces. The space $\mathcal{D}_{k}^{\prime}$ is the celebrated space of Schwartz distributions for $k=0$ and $U=\mathbb{R}^{n}$. Let $\mathcal{B}_{k}^{r}$ be the Fréchet space of $k$-forms whose Lie derivatives are bounded up to order $r$, and $\mathcal{B}_{k}=\lim _{\leftarrow} \mathcal{B}_{k}^{r}$. 
Most of this paper concerns the space ' $\mathcal{B}_{k}$ which is now well developed ( [5-10]). First appearing in [5] is a constructive, geometric definition using "difference chains," which does not rely on a space of differential forms. (See also 12 for an elegant exposition. An earlier approach based on polyhedral chains can be found in [4.) We use an equivalent definition below using differential forms $\mathcal{B}_{k}$ and the space of pointed $k$-chains $\mathcal{P}_{k}$ which has the advantage of of brevity.

We can write an element $A \in \mathcal{P}_{k}(U)$ as a formal sum $A=\sum_{i=1}^{s}\left(p_{i} ; \alpha_{i}\right)$ where $p_{i} \in U$, and $\alpha_{i} \in$ $\Lambda_{k}\left(T_{p} U\right)$. Define a family of norms on $\mathcal{P}_{k}$,

$$
\|A\|_{B^{r}}=\sup _{0 \neq \omega \in \mathcal{B}_{k}^{r}} \frac{f_{A} \omega}{\|\omega\|_{C^{r}}}
$$

for $r \geq 0$, where $f_{A} \omega:=\sum_{i=1}^{s} \omega\left(p_{i} ; \alpha_{i}\right)$. Let $\hat{\mathcal{B}}_{k}^{r}$ denote the Banach space on completion, and $\hat{\mathcal{B}}_{k}=\lim _{\longrightarrow} \hat{\mathcal{B}}_{k}^{r}$ the inductive limit. We endow $\hat{\mathcal{B}}_{k}$ with the inductive limit topology $\tau$. Since the norms are decreasing, the Banach spaces form an increasing nested sequence. As of this writing, it is unknown whether $\hat{\mathcal{B}}_{k}$ is complete. Since $\hat{\mathcal{B}}_{k}$ is a locally convex space, we may take its completion (see Schaefer [13], p. 17) in any case, and denote the resulting space by ${ }^{\prime} \mathcal{B}_{k}(U)$. Elements of ' $\mathcal{B}_{k}(U)$ are called "differential $k$-chains 11 in $U . "$

The reader might ask how ${ }^{\prime} \mathcal{B}_{k}(U)$ relates to the space $\mathcal{B}_{k}^{\prime}(U)$ of currents, endowed with the strong dual topology. We prove below that ${ }^{\prime} \mathcal{B}_{k}(U)$ is not generally reflexive. However, under the canonical inclusion $u:{ }^{\prime} \mathcal{B}_{k}(U) \rightarrow \mathcal{B}_{k}^{\prime}(U)$, this subspace of currents is closed under the primitive and fundamental operators used in the Cartan calculus (see Harrison [10]). Thus, differential chains form a distinguished subspace of currents that is constructively defined and approximable by pointed chains. That is, while $\mathcal{P}_{k}$ is dense in $\mathcal{B}_{k}^{\prime}$ in the weak topology, $\mathcal{P}_{k}$ is in fact dense in ' $\mathcal{B}_{k}$ in the strong topology. More specifically, when $\mathcal{B}_{k}^{\prime}(U)$ is given the strong topology, the space $u\left({ }^{\prime} \mathcal{B}_{k}(U)\right)$ equipped with the subspace topology is topologically isomorphic to ${ }^{\prime} \mathcal{B}_{k}(U)$. Thus in the case of differential forms $\mathcal{B}_{k}\left(\mathbb{R}^{n}\right)$ question (i) has a negative, and (ii) has an affirmative answer.

\section{Topological Properties}

Proposition 2.0.1. $\hat{\mathcal{B}}_{k}$ is an ultrabornological, bornological, barreled, (DF), Mackey, Hausdorff, and locally convex space. ' $\mathcal{B}_{k}$ is barreled, $(D F)$, Mackey, Hausdorff and locally convex.

Proof. By definition, the topology on $\hat{\mathcal{B}}_{k}$ is locally convex. We showed that $\hat{\mathcal{B}}_{k}$ is Hausdorff in [10]. According to K othe [11, p. 403, a locally convex space is a bornological ( $D F$ ) space if and only if it is the inductive limit of an increasing sequence of normed spaces. It is ultrabornological if it is the inductive limit of Banach spaces. Therefore, $\hat{\mathcal{B}}_{k}$ is an ultrabornological $(D F)$-space.

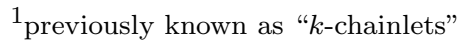


Every inductive limit of metrizable convex spaces is a Mackey space (Robertson [1] p. 82). Therefore, $\hat{\mathcal{B}}_{k}$ is a Mackey space. It is barreled according to Bourbaki [2] III 45, 19(a).

The completion of any locally convex Hausdorff space is also locally convex and Hausdorff. The completion of a barreled space is barreled by Schaefer [13, p. 70 exercise 15 and the completion of a $(D F)$-space is $(D F)$ by [13] p.196 exercise $24(\mathrm{~d})$. But the completion of a bornological space may not be bornological (Valdivia [15])

Theorem 2.0.2 (Characterization 1). The space of differential chains ' $\mathcal{B}_{k}$ is the completion of pointed chains $\mathcal{P}_{k}$ given the Mackey topology $\tau\left(\mathcal{P}_{k}, \mathcal{B}_{k}\right)$.

Proof. Since $\left(\mathcal{P}_{k}, \mathcal{B}_{k}\right)$ is a dual pair, the Mackey topology $\tau\left(\mathcal{P}_{k}, \mathcal{B}_{k}\right)$ is well-defined (Robertson [1]). This is the finest locally convex topology on $\mathcal{P}_{k}$ such that the continuous dual $\mathcal{P}_{k}^{\prime}$ is equal to $\mathcal{B}_{k}$.

Let $\left.\tau\right|_{\mathcal{P}_{k}}$ be the subspace topology on pointed chains $\mathcal{P}_{k}$ given the inclusion of $\mathcal{P}_{k}$ into $\hat{\mathcal{B}}_{k}$. By (2.0.1), the topology on $\hat{\mathcal{B}}_{k}$ is Mackey; explicitly it is the topology of uniform convergence on relatively $\sigma\left(\mathcal{B}_{k}, \hat{\mathcal{B}_{k}}\right)$-compact sets where $\sigma\left(\mathcal{B}_{k}, \hat{\mathcal{B}_{k}}\right)$ is the weak topology on the dual pair $\left(\mathcal{B}_{k}, \hat{\mathcal{B}_{k}}\right)$. But then $\left.\tau\right|_{\mathcal{P}_{k}}$ is the topology of uniform convergence on relatively $\sigma\left(\mathcal{B}_{k}, \mathcal{P}_{k}\right)$-compact sets, which is the same as the Mackey topology $\tau$ on $\mathcal{P}_{k}$. Therefore, $\left.\tau\right|_{\mathcal{P}_{k}}=\tau\left(\mathcal{P}_{k}, \mathcal{B}_{k}\right)$.

\section{Relation of Differential Chains to Currents}

The Fréchet topology $F$ on $\mathcal{B}=\mathcal{B}_{k}$ is determined by the seminorms $\|\omega\|_{C^{r}}=\sup _{J \in Q^{r}} \omega(J)$, where $Q^{r}$ is the image of the unit ball in $\hat{\mathcal{B}}_{k}^{r}$ via the inclusion $2 \hat{\mathcal{B}}_{k}^{r} \hookrightarrow \hat{\mathcal{B}}_{k}$.

Lemma 3.0.3. $\left(\mathcal{B}, \beta\left(\mathcal{B},{ }^{\prime} \mathcal{B}\right)\right)=(\mathcal{B}, F)$.

Proof. First, we show $F$ is coarser than $\beta\left(\mathcal{B},{ }^{\prime} \mathcal{B}\right)$. It is enough to show that the Fréchet seminorms are seminorms for $\beta$. For every form $\omega \in \mathcal{B}_{k}$ there exists a scalar $\lambda_{\omega, r}$ such that $\left\|\lambda_{\omega, r} \omega\right\|_{C^{r}} \leq 1$. In other words, $Q^{r 0}$, the polar of $Q^{r}$ in $\mathcal{B}_{r}$, is absorbent and hence $\|\cdot\|_{C^{r}}$ is a seminorm for $\beta$.

On the other hand, $\beta\left(\mathcal{B},{ }^{\prime} \mathcal{B}\right)$, as the strong dual topology of a $(D F)$ space, is also Fréchet, and hence by [1] the two topologies are equal.

Theorem 3.0.4. The vector space ${ }^{\prime} \mathcal{B}\left(\mathbb{R}^{n}\right)$ is a proper subspace of the vector space $\mathcal{B}^{\prime}\left(\mathbb{R}^{n}\right)$.

\footnotetext{
${ }^{2}$ The authors show this inclusion is compact in a sequel.
} 
Remark: 'B $\mathcal{B}$ and $\mathcal{B}$ are barreled. Thus semi-reflexive and reflexive are identical for both spaces.

Proof. Schwartz defines $(\mathcal{B})$ in $\S 8$ on page 55 of [14 as the space of functions with all derivatives bounded on $\mathbb{R}^{n}$, and endows it with the Fréchet space topology, just as we have done. He writes on page 56, "( $\left.\mathcal{D}_{L^{1}}\right),(\dot{\mathcal{B}}),(\mathcal{B})$, ne sont pas réflexifs." Suppose ' $\mathcal{B}_{k}\left(\mathbb{R}^{n}\right)$ is reflexive. By Lemma 3.0.3 the strong dual of ${ }^{\prime} \mathcal{B}_{k}\left(\mathbb{R}^{n}\right)$ is $\left(\mathcal{B}\left(\mathbb{R}^{n}\right), F\right)$. This implies that $\left(\mathcal{B}\left(\mathbb{R}^{n}\right), F\right)$ is reflexive, contradicting Schwartz.

We immediately deduce:

Theorem 3.0.5. The space ' $\mathcal{B}_{k}$ carries the subspace topology of $\mathcal{B}_{k}^{\prime}$, where $\mathcal{B}_{k}^{\prime}$ is given the strong topology $\beta\left(\mathcal{B}_{k}^{\prime}, \mathcal{B}_{k}\right)$.

Corollary 3.0.6 (Characterization 2). The topology $\tau\left(\mathcal{P}_{k}, \mathcal{B}_{k}\right)$ on $\mathcal{P}_{k}$ is the subspace topology on $\mathcal{P}_{k}$ considered as a subspace of $\left(\mathcal{B}_{k}^{\prime}, \beta\left(\mathcal{B}_{k}^{\prime}, \mathcal{B}_{k}\right)\right)$.

Remarks 3.0.7. We immediately see that $\mathcal{P}_{k}$ is not dense in $\mathcal{B}_{k}^{\prime}$. Compare this to the BanachAlaoglu theorem, which implies $\mathcal{P}_{k}$ is weakly dense in $\mathcal{B}_{k}^{\prime}$, whereas $\mathcal{P}_{k}$ is strongly dense in ' $\mathcal{B}_{k}$.

In fact, Corollaries 2.0.2 and 3.0.6 suggest a more general statement: let $E$ be an arbitrary locally convex topological vector space. Elements of $E$ will be our "generalized forms." Let $P$ be the vector space generated by extremal points of open neighborhoods of the origin in $E^{\prime}$ given the strong topology. These will be our "generalized pointed chains." Then $(P, E)$ forms a dual pair and so we may put the Mackey topology $\tau$ on $P$. We may also put the subspace topology $\sigma$ on $P$, considered as a subspace of $E^{\prime}$ with the strong topology. We ask the following questions: under what conditions on $E$ will $\tau=\sigma$ ? Under what conditions will $P$ be strongly dense in $E^{\prime}$ ? What happens when we replace $\mathcal{B}$ with $\mathcal{D}, \mathcal{E}$ or $\mathcal{S}$, the Schwartz space of forms rapidly decreasing at infinity?

Theorem 3.0.8. The space ' $\mathcal{B}_{k}\left(\mathbb{R}^{n}\right)$ is not nuclear, normable, metrizable, Montel, or reflexive.

Proof. The fact that $\hat{\mathcal{B}}_{k}\left(\mathbb{R}^{n}\right)$ is not reflexive follows from Theorem 3.0.4, It is well known that $\mathcal{B}_{k}\left(\mathbb{R}^{n}\right)$ is not a normable space. Therefore, ${ }^{\prime} \mathcal{B}_{k}\left(\mathbb{R}^{n}\right)$ is not normable. If $E$ is metrizable and $(D F)$, then $E$ is normable (see p. 169 of Grothendieck [3]). Since ${ }^{\prime} \mathcal{B}_{k}\left(\mathbb{R}^{n}\right)$ is a $(D F)$ space, it is not metrizable. If a nuclear space is complete, then it is semi-reflexive, that is, the space coincides with its second dual as a set of elements.

Therefore, ' ${ }^{\prime} \mathcal{B}_{k}\left(\mathbb{R}^{n}\right)$ is not nuclear. Since all Montel spaces are reflexive, we know that $\hat{\mathcal{B}}_{k}\left(\mathbb{R}^{n}\right)$ is not Montel. 


\section{INDEPENDENT CHARACTERIZATION}

We can describe our topology $\tau\left(\mathcal{P}_{k}, \mathcal{B}_{k}\right)$ on $\mathcal{P}_{k}$ in another non-constructive manner for $U$ open in $\mathbb{R}^{n}$, this time without reference to the space $\mathcal{B}$.

Theorem 4.0.9 (Characterization 3). The topology $\tau\left(\mathcal{P}_{k}, \mathcal{B}_{k}\right)$ is the finest locally convex topology $\mu$ on $\mathcal{P}_{k}$ such that

(1) bounded mappings $\left(\mathcal{P}_{k}, \mu\right) \rightarrow F$ are continuous whenever $F$ is locally convex;

(2) $K^{0}=\left\{(p ; \alpha) \in \mathcal{P}_{k}:\|\alpha\|=1\right\}$ is bounded in $\left(\mathcal{P}_{k}, \mu\right)$, where $\|\alpha\|$ is the mass norm of $\alpha \in \Lambda_{k}\left(\mathbb{R}^{n}\right)$;

(3) $P_{v}:\left(\mathcal{P}_{k}, \mu\right) \rightarrow \overline{\left(\mathcal{P}_{k}, \mu\right)}$ given by $P_{v}(p ; \alpha):=\lim _{t \rightarrow 0}(p+v ; \alpha / t)-(p ; \alpha / t)$ is well-defined and bounded for all vectors $v \in \mathbb{R}^{n}$.

Proof. A l.c.s. $E$ is bornological if and only if bounded mappings $S: E \rightarrow F$ are continuous whenever $F$ is locally convex. Any subspace of a bornological space is bornological. So by proposition 2.0.1, $\tau$ satisfies (1). Properties (2) and (3) are established in Harrison [4, 10].

Now suppose $\mu$ satisfies (1)-(3). Suppose $\omega \in\left(\mathcal{P}_{k}, \mu\right)^{\prime}$. Then

$$
\begin{aligned}
\omega P_{v}(p ; \alpha) & =\omega\left(\lim _{t \rightarrow 0}(p+t v ; \alpha / t)-(p ; \alpha / t)\right)=\lim _{t \rightarrow 0} \omega((p+t v ; \alpha / t)-(p ; \alpha / t)) \\
& =\lim _{t \rightarrow 0} \omega(p+t v ; \alpha / t)-\omega(p ; \alpha / t)=L_{v} \omega(p ; \alpha),
\end{aligned}
$$

where $L_{v}$ is the Lie derivative of $\omega$. Since $\omega$ is continuous and $K^{0}$ is bounded, it follows that $\omega\left(K^{0}\right)$ is bounded in $\mathbb{R}$. (see [2] III.11 Proposition 1(iii)). Similarly, $K^{r}=P_{v}\left(K^{r-1}\right)$ is bounded implies $\omega\left(K^{r}\right)$ is bounded. It follows that $\omega \in \mathcal{B}_{k}$. Hence $\left(\mathcal{P}_{k}, \mu\right)^{\prime} \subset \mathcal{B}_{k}$. Now $\mu$ is Mackey since it is bornological. Since $\left(\mathcal{P}_{k}, \mu\right)^{\prime} \subset\left(\mathcal{P}_{k}, \tau\right)^{\prime}$ and $\tau$ is also Mackey, we know that $\tau$ is finer than $\mu$ by the Mackey-Arens theorem.

Example 4.0.10. Let $\|A\|_{\natural}=\lim _{\rightarrow}\|A\|_{B^{r}}$. This is a norm on pointed chains (Harrison [4]). The Banach space $(\mathcal{P}, \emptyset)$ satisfies $(1)-(3)$. The topology $\emptyset$ is strictly coarser than $\tau$ since $(\mathcal{P}, t)^{\prime}=\mathcal{B}$ and $(\mathcal{P}, \emptyset)^{\prime}$ is the space of differential forms with a uniform bound on all directional derivatives.

\section{REFERENCES}

[1] Alexander and Wendy Robertson. Topological Vector Spaces. Cambridge University Press, Cambridge, 1964.

[2] Nicolas Bourbaki. Elements of Mathematics: Topological Vector Spaces. Springer-Verlag, Berlin, 1981.

[3] Alexander Grothendieck. Topological Vector Spaces. Gordon Breach, 1973. 
[4] J. Harrison. Differential complexes and exterior calculus.

[5] Jenny Harrison. Stokes' theorem on nonsmooth chains. Bulletin of the American Mathematical Society, 29:235$242,1993$.

[6] Jenny Harrison. Continuity of the integral as a function of the domain. Journal of Geometric Analysis, 8(5):769$795,1998$.

[7] Jenny Harrison. Isomorphisms of differential forms and cochains. Journal of Geometric Analysis, 8(5):797-807, 1998.

[8] Jenny Harrison. Geometric Hodge star operator with applications to the theorems of Gauss and Green. Mathematical Proceedings of the Cambridge Philosophical Society, 140(1):135-155, 2006.

[9] Jenny Harrison. Geometric Poincare Lemma. submitted, March 2010.

[10] Jenny Harrison. Operator calculus - the exterior differential complex. submitted, March 2010.

[11] Gottfried Köthe. Topological Vector Spaces, volume I. Springer-Verlag, Berlin, 1966.

[12] Harrison Pugh. Applications of differential chains to complex analysis and dynamics. Harvard senior thesis, 2009.

[13] Helmut Schaefer. Topological Vector Spaces. McMillan Company, 1999.

[14] Laurent Schwartz. Théorie des Distributions, Tome II. Hermann, Paris, 1954.

[15] Manuel Valdivia. On the completion of a bornological space. Arch. Math. Basel, 29:608-613, 1977. 